\title{
Anisotropy of Elementary Particle Fluxes in Primary Cosmic Rays Measured with the Alpha Magnetic Spectrometer on the ISS
}

\author{
M. A. Velasco ${ }^{a, *}$ and J. Casaus, C. Mañá, M. Molero ${ }^{a}$ \\ ${ }^{a}$ Centro de Investigaciones Energéticas, Medioambientales y Tecnológicas (CIEMAT), \\ Avda. Complutense 40, Madrid, Spain \\ E-mail: MiguelAngel.Velasco@Ciemat.es
}

\begin{abstract}
A measurement of the cosmic ray anisotropy on the arrival directions of elementary particles (electrons, positrons and protons) has been performed in galactic coordinates by the Alpha Magnetic Spectrometer onboard the International Space Station. The analysis is based on the sample of events collected in the first 8.5 years of data taking. The results are consistent with isotropy for all cosmic ray species and upper limits on the dipole amplitude have been computed. In particular, $95 \%$ credible interval upper limits of $\delta<1.7 \%$ and $\delta<0.37 \%$ are obtained for positrons and electrons, respectively, above $16 \mathrm{GeV}$. On the other hand, the upper limit to the dipole anisotropy of protons above $200 \mathrm{GV}$ is found to be $\delta<0.35 \%$.
\end{abstract}

40th International Conference on High Energy physics - ICHEP2020

July 28 - August 6, 2020

Prague, Czech Republic (virtual meeting)

\footnotetext{
${ }^{*}$ Speaker
} 


\section{Introduction}

In the last years, the Alpha Magnetic Spectrometer (AMS-02) onboard the International Space Station (ISS) has provided precise measurements of cosmic ray fluxes, which have revealed many unexpected features that cannot be fully explained within the current understanding of cosmic rays acceleration and propagation.

On the one hand, the positron spectrum [1] shows a significant excess above $\sim 25 \mathrm{GeV}$ followed by a sharp dropoff above $\sim 284 \mathrm{GeV}$ with a finite exponential energy cutoff at $\sim 810 \mathrm{GeV}$ established at more than $4 \sigma$. The observation is not consistent with a pure secondary origin of cosmic ray positrons and, in many models, the inclusion of a source term, either from dark matter annihilation or astrophysical sources [2, 3], is required to explain the origin of the excess. Furthermore, the electron spectrum [4] exhibits a significant excess above $\sim 42 \mathrm{GeV}$ compared to the lower energy trends, which is well described by the sum of two power law contributions in the entire energy range. Contrary to the positron flux, the electron flux does not have an energy cutoff below $1.9 \mathrm{TeV}$, which suggests a different origin for high energy electrons and positrons.

On the other hand, the proton [5] spectrum shows a deviation from a single power law, with a progressive hardening of the spectral index above $\sim 200 \mathrm{GV}$. The origin of this spectral feature may be due to the existence of nearby sources in the Galaxy contributing at high rigidities, or may require a modification of the cosmic ray transport models $[6,7]$.

In all cases, the existence of nearby compact sources of cosmic rays may induce a sizable anisotropy in their arrival directions. Thus, the measurement of the cosmic ray anisotropy is a complementary study to characterize the observed spectral features and may help to understand their origin.

\section{The AMS-02 detector}

AMS-02 is a multipurpose particle physics detector designed to carry out accurate measurements of cosmic ray charged particles in the GeV-TeV range. It was installed on 19 May 2011 onboard the International Space Station and it continues taking data steadily since then. So far, AMS-02 has collected more than 160 billion events of galactic cosmic rays in a long term mission, which is supposed to continue during ISS lifetime until 2028.

The detector consists of nine layers of precision silicon tracker (STD), with an inner tracker (L2-L8) inside a permanent magnet and two outer layers (L1 and L9); a transition radiation detector (TRD); four planes of time of flight counters (TOF); an array of anti-coincidence counters (ACC) surrounding the inner tracker; a ring imaging Čerenkov detector (RICH); and an electromagnetic calorimeter (ECAL). More details on the sub-detectors can be found in [8].

\section{Data selection}

Positron and electron events are selected by requiring a track in the TRD and in the tracker, a cluster of hits in the ECAL, and a measured velocity $\beta \sim 1$ in the TOF consistent with a downwardgoing $Z=1$ relativistic particle. Positrons and electrons are separated from protons by means of a selection based on a cut on the ECAL estimator and a template fit to the TRD response. The 
final sample contains $1.7 \times 10^{5}$ positrons and $2.3 \times 10^{6}$ electrons above $16 \mathrm{GeV}$. For the anisotropy analysis, events are grouped into 5 cumulative energy ranges from 16 to $350 \mathrm{GeV}$ according to their measured energy in the ECAL, with minimum energies: 16, 25, 40, 65 and $100 \mathrm{GeV}$ respectively.

Preselected proton events include downward-going particles with velocity measured by four TOF planes and charge measurement consistent with the corresponding to each particle species. Events reconstructed by the 7 inner layers, passing through the two outer layers L1 and L9, and satisfying additional track quality criteria are finally selected. Above $18 \mathrm{GV}$, the final proton sample includes $1.4 \times 10^{8}$ events. The anisotropy analysis is performed on 9 cumulative rigidity ranges of minimum rigidity: $18,30,45,80,150,200,300,500,1000 \mathrm{GV}$.

In addition, to select only primary cosmic rays well above the geomagnetic cutoff, the measured rigidity is required to be above the maximum geomagnetic cutoff within the AMS field of view.

\section{Methodology}

The analysis of anisotropies in a sample is performed by comparing the observed distribution of arrival directions in galactic coordinates, $(l, b)$, with a reference map. Skymaps for both sample of study and reference are built using HEALPix scheme [9], which ensures a pixelization with an equal area isolatitude subdivisions of the sphere.

The reference map for anisotropy studies describes the directional response of the detector to an isotropic flux, and its computation requires a detailed understanding of experimental effects. In particular, a geographical variation of detector efficiencies may project onto the galactic coordinate system and induce an spurious signal if not accounted. Therefore, a precise knowledge of these variations is needed to disentagle a possible physical signal from the effects related to the performance of the detector. A systematic procedure, valid for all cosmic ray species, has been developed to obtain these isotropic skymaps [10].

A likelihood fit is used to compare the distribution of events under study and the reference map, and takes into account the differences in the exposure for different energies or rigidities due to geomagnetic cutoff variation along the orbit. This procedure proved to be stable against different map resolutions and sample statistics. A spherical harmonics expansion in terms of multipolar coefficients, $a_{\ell m}$, is used to described the directional dependence of the flux:

$$
\Phi(l, b)=\Phi_{0}\left(1+\sum_{\ell>0} \sum_{m=-\ell}^{m=+\ell} a_{\ell m} Y_{\ell m}(l, b)\right)
$$

At first order, $\ell=1$, the dipole is fully described by three orthonormal functions corresponding to three orthogonal axes: $Y_{1+1}$ is aligned with the forward-backward direction, pointing to the galactic center; $Y_{1+0}$ is aligned with the north-south direction, pointing to the north galactic pole; and $Y_{1-1}$ is aligned with the east-west direction, contained in the galactic plane and completes the right-handed coordinate system. Dipole components in each direction are defined as

$$
\rho_{\mathrm{EW}}=\sqrt{\frac{3}{4 \pi}} a_{1-1} \quad ; \quad \rho_{\mathrm{NS}}=\sqrt{\frac{3}{4 \pi}} a_{1+0} \quad ; \quad \rho_{\mathrm{FB}}=\sqrt{\frac{3}{4 \pi}} a_{1+1}
$$

Finally, the dipole amplitude, which quantifies the asymmetry between the maximum and minimum of the flux, is

$$
\delta=\frac{\Phi_{\max }-\Phi_{\min }}{\Phi_{\max }+\Phi_{\min }}=\sqrt{\rho_{\mathrm{EW}}^{2}+\rho_{\mathrm{NS}}^{2}+\rho_{\mathrm{FB}}^{2}}
$$




\section{Positron and electron anisotropy}

The measurement of the anisotropy in the arrival directions of the positrons and electrons collected by AMS-02 in the first 8.5 years of data taking revealed no deviation from isotropy. Consequently, upper limits on the dipole amplitude at the $95 \%$ credible interval (C.I.) were computed for the different energy ranges of the analysis (figures 1 and 2 ). In particular, the upper limits for positrons and electrons above $16 \mathrm{GeV}$ are found to be $\delta<1.7 \%$ and $\delta<0.37 \%$, respectively.

$\rho_{\mathrm{EW}}$

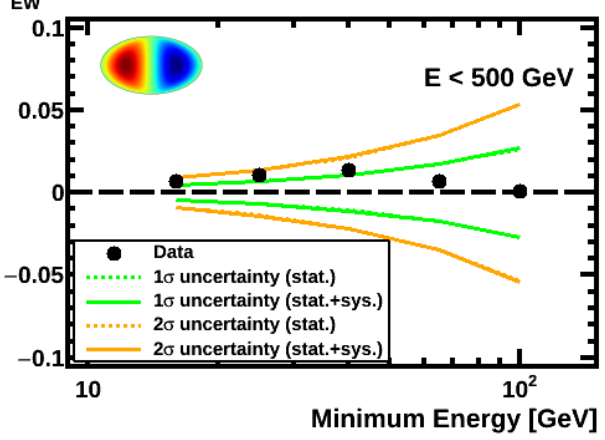

$\rho_{\mathrm{FB}}$

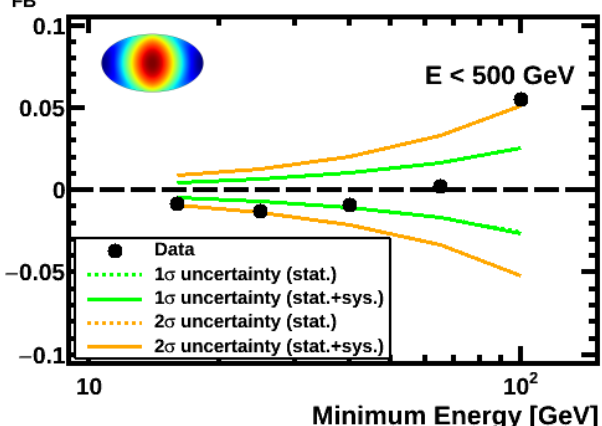

$\rho_{\text {NS }}$
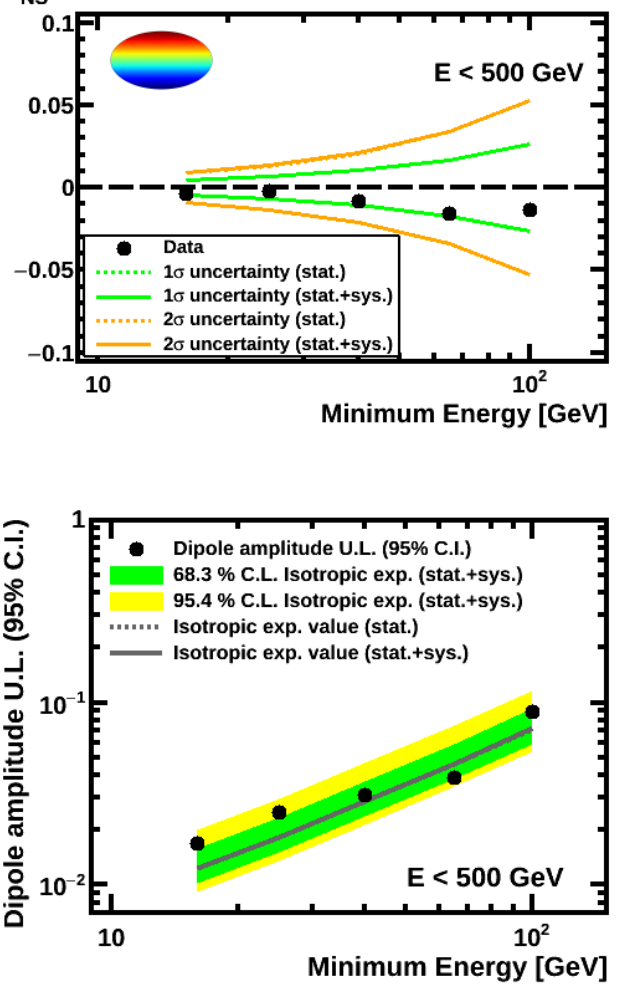

Figure 1: Amplitudes of the dipole components of the positron anisotropy: east-west (top left), north-south (top right) and forward-backward (bottom left) as a function of the minimum energy. 95\% C.I. upper limits on the dipole amplitude (bottom right). Bands corresponding to isotropic expectation at $68.3 \%$ (green) and 95.4\% C.I. (yellow) are also displayed. 


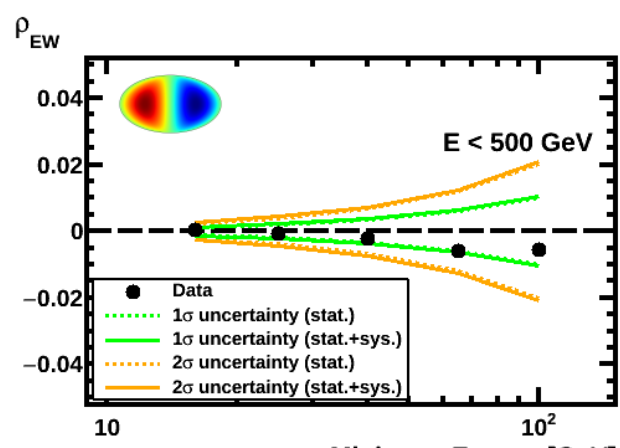

Minimum Energy [GeV]

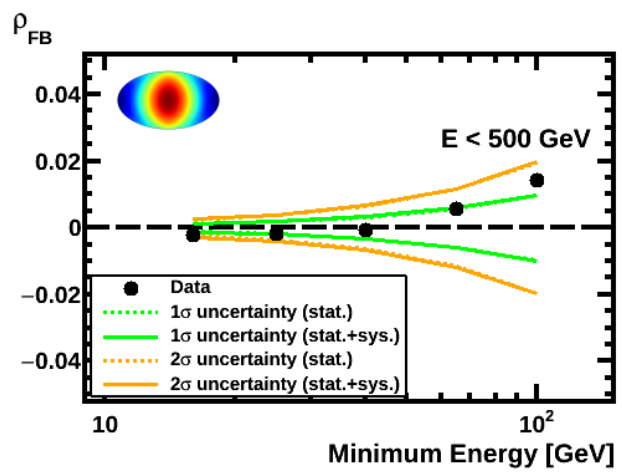

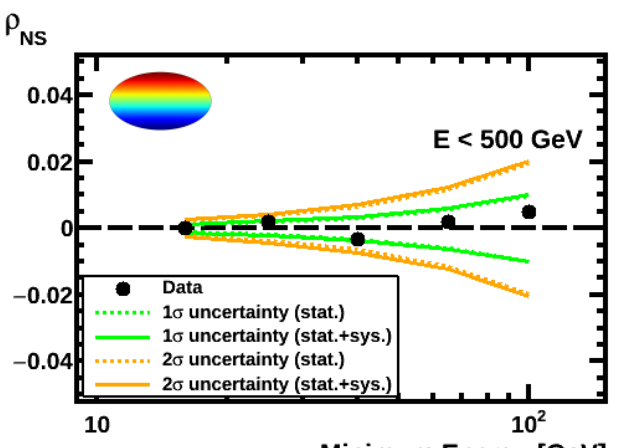

Minimum Energy [GeV]

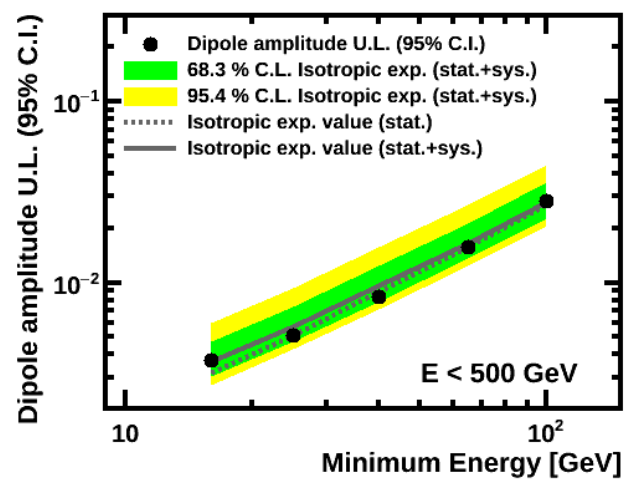

Figure 2: Amplitudes of the dipole components of the electron anisotropy: east-west (top left), north-south (top right) and forward-backward (bottom left) as a function of the minimum energy. 95\% C.I. upper limits on the dipole amplitude (bottom right). Bands corresponding to isotropic expectation at $68.3 \%$ (green) and $95.4 \%$ C.I. (yellow) are also displayed.

\section{Proton anisotropy}

The results of the anisotropy analysis on the 8.5 -years proton sample were found to be consistent with isotropy. Therefore, 95\% C.I. upper limits on the dipole amplitude were calculated for each rigidity range (figure 3). In particular, the proton anisotropy is dominated by statistics above $70 \mathrm{GV}$ and systematics limit the measurement at low rigidities to $0.1 \%$. Finally, for rigidities above 200 $\mathrm{GV}$, the upper limits are set to $\delta<0.35 \%$.

\section{Conclusions}

The measurement of the anisotropy in the arrival directions of cosmic ray electrons, positrons and protons has been carried out by the AMS-02 experiment on the ISS. The results in galactic coordinates show no deviation from isotropy and 95\% C.I. upper limits on the dipole amplitude were obtained. In particular, limits of $\delta<1.7 \%$ and $\delta<0.37 \%$ have been set for positrons and electrons, respectively, above $16 \mathrm{GeV}$. On the other hand, the limits to the dipole amplitude on protons were found to be $\delta<0.35 \%$ above $200 \mathrm{GV}$. 

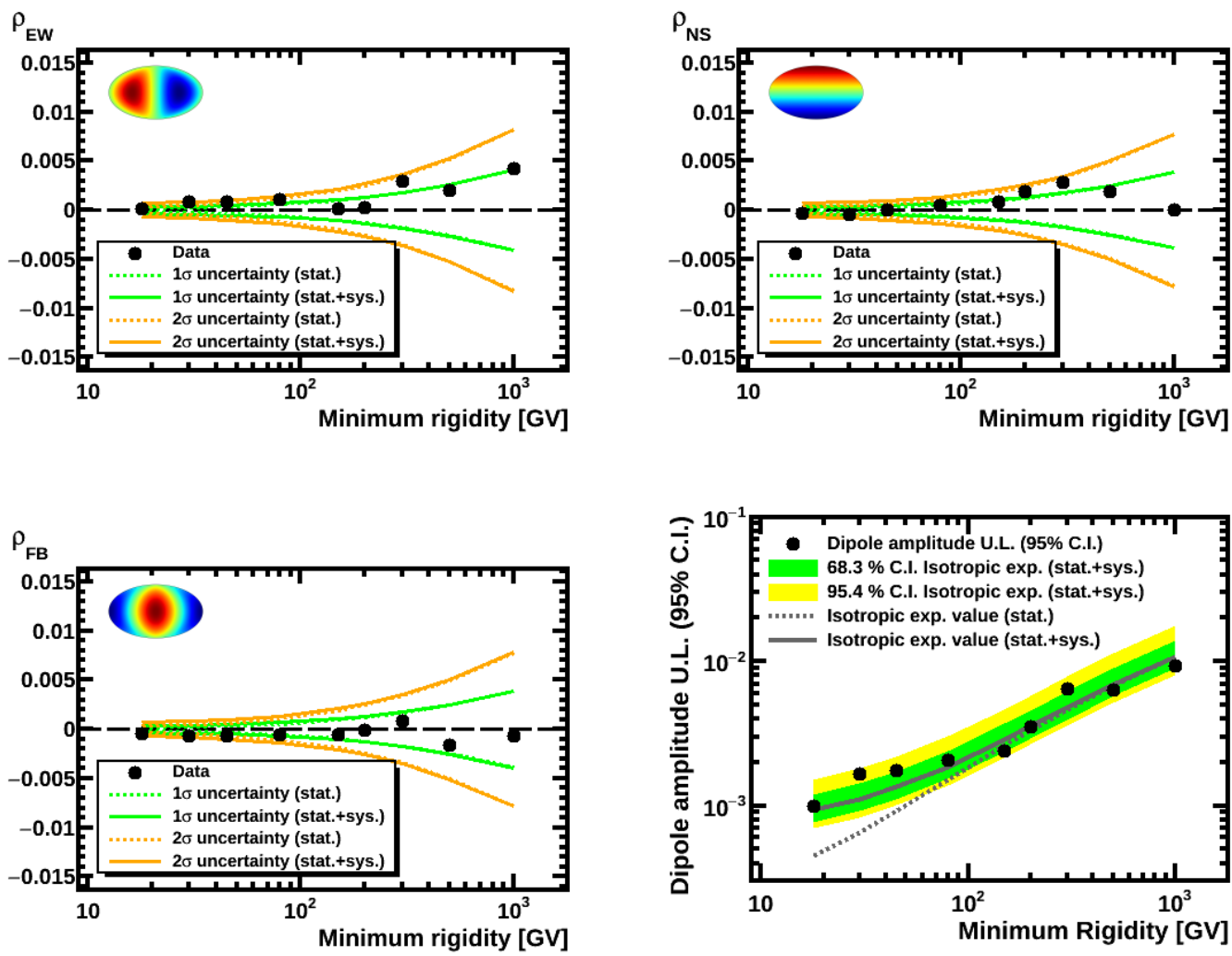

Figure 3: Amplitudes of the dipole components of the proton anisotropy: east-west (top left), north-south (top right) and forward-backward (bottom left) as a function of the minimum rigidity. 95\% C.I. upper limits on the dipole amplitude (bottom right). Bands corresponding to isotropic expectation at $68.3 \%$ (green) and $95.4 \%$ C.I. (yellow) are also displayed.

\section{References}

[1] M. Aguilar et al. (AMS Collaboration) Phys. Rev. Lett. 122041102 (2019)

[2] M. Boudaud et al. A\&A 575 A67 (2015)

[3] M. Di Mauro, F. Donato, N. Fornengo and A. Vittino JCAP 1605031 (2016)

[4] M. Aguilar et al. (AMS Collaboration) Phys. Rev. Lett. 122101101 (2019)

[5] M. Aguilar et al. (AMS Collaboration) Phys. Rev. Lett. 114171103 (2015)

[6] G. Bernard et al. A\&A 555 A48 (2013)

[7] R. Aloisio, P. Blasi and P. D. Serpico A\&A 583 A95 (2015)

[8] M. Aguilar et al. (AMS Collaboration) Phys. Rev. Lett. 110141102 (2013)

[9] K. M. Górski et al. Astrophys.J. 622759 (2005)

[10] M. A. Velasco, J. Casaus and C. Mañá PoS ICRC2017 196 (2018) 